\title{
Relationship between Elastic Recovery and Macroscopic- and Microscopic- Structures of Hydrogenated Polybutadiene Films
}

\author{
Akira TANAKA, Yasuhiro SAIHARA, and Shigeharu ONOGI* \\ Department of Polymer Chemistry, Faculty of Engineering, Kyoto University, \\ Yoshida, Sakyo-ku, Kyoto 606, Japan.
}

(Received January 11, 1980)

\begin{abstract}
Hydrogenated polybutadiene films, especially those that are quenched, exhibited marked elastic recovery even under fairly large strain. In order to clarify the relationship between elastic recovery and macro- and microscopic-structure of the hydrogenated polybutadiene films, Xray diffraction and optical properties such as birefringence and infrared dichroism were measured simultaneously and mechanical properties were also studied. The results were compared with those for low-density polyethylene films, having chemical structures similar to the hydrogenated polybutadiene films but which do not exhibit marked elastic recovery. The elastic properties of the hydrogenated polybutadiene films are attributed to their loose spherulitic structures which allow crystallites to orient in a reversible manner. On the other hand, spherulitic structures of low-density polyethylene films are so tight that crystallites cannot orient under a relatively large strain without breaking the spherulites.
\end{abstract}

KEY WORDS Rheo-optics / Elastic Recovery / Deformation Mechanism / Macroscopic Structure / Microscopic Structure / Hydrogenated Polybutadiene / Polyethylene /

Hydrogenated polybutadiene (HPB) should have a chemical structure the same as that of linear polyethylene, if the polymerization of butadiene proceeds by the 1, 4 addition and the resulting polybutadiene is completely hydrogenated. However, HPB films show fairly different properties from those of the usual polyethylene films. The most remarkable difference is that the HPB film, especially when quenched exhibits a marked elastic recovery even under a fairly large strain. Also, HPB films are transparent. Furthermore, a HPB film can be formed at lower temperatures as compared with polyethylene having a comparable molecular weight. To clarify the relationship between the elastic recovery and the macro- and microscopicstructure of HPB, the viscoelastic and rheo-optical properties were measured and compared with those for low-density polyethylene films.

* To whom all communication should be addressed.

\section{EXPERIMENTAL}

Samples

The hydrogenated polybutadiene (HPB) used in this study was provided by Prof. W. W. Graessley (Northwestern University). This material was prepared by hydrogenation of polybutadiene that had been made by anionic polymerization. The polymerization of butadiene was carried out in toluene, using butyllithium as the initiator according to the method reported by Hsieh. ${ }^{1}$ The hydrogenation of polybutadiene was performed with a coordinatetype catalyst at $50^{\circ} \mathrm{C}$ and $3.5 \mathrm{~atm}$ hydrogen pressure after the method reported by Falk. ${ }^{2}$ The catalyst was made by the reaction of butyllithium with transition-metal salts of 2-ethyl caproic acid.

According to a private communication, ${ }^{3}$ the weight-average molecular weight of the polybutadiene (PBD), being an intermediate product, is 558,000 and its molecular weight distribution is rather narrow, as is shown by the ratio of the weight-average molecular weight to the numberaverage molecular weight of 1.6. Thus, the weightaverage molecular weight of the resulting HPB is 
estimated to be 579,000 , and its molecular weight distribution must be narrow of the same order of the original PBD.

To prepare HPB films, the material was pressed between two aluminum plates in a laboratory press at $130^{\circ} \mathrm{C}$ and $100 \mathrm{~kg} \mathrm{~cm}^{-2}$ for $10 \mathrm{~min}$. Then, the material, while situated between the aluminum plates, was plunged into an ice-water bath. The film specimen thus prepared is referred to as HPBD-Q. Another kind of film, referred to as HPBD-A was also prepared; the material which was melt-pressed under the same conditions as above was gradually cooled to room temperature.

Commercial low-density polyethylene, UBE B$128\left(M_{n} \fallingdotseq 27,000, M_{w} / M_{n} \fallingdotseq 8.8\right)$, produced by UBE Industries Ltd. was used for comparison. Films of this material were also prepared under almost the same conditions above. In this case, however, the temperature of the hot press was kept at $140^{\circ} \mathrm{C}$. Films thus obtained are referred to as LDPE-Q and LDPE-A.

\section{Characterization of the Sample Films}

Microscopic Characteristic. The crystal form of HPB films was investigated by the X-ray diffraction method. The X-ray diffraction pattern indicates that the crystal form is orthorhombic in both the quenched and annealed films. This crystal form is the same as that observed for bulk-crystallized polyethylene.

The infrared spectrum of HPBD-Q film is shown in Figure 1. The HPBD-A film gave a very similar spectrum. These spectra are very similar to that of polyethylene film but show some discrepancies. First, the peaks at $1378 \mathrm{~cm}^{-14}$ and $770 \mathrm{~cm}^{-15}$ assigned to methyl- and ethyl-branches are clearly recognized. By the method of Shirayama et al., ${ }^{6}$ the number of ethyl-branches was estimated to be about 2.5 per 100 carbon atoms. This value is fairly larger than that for the polyethylene as shown in Table I. This might originate from a small amount of $1-2$ addition as well as $1-4$ addition in the process of

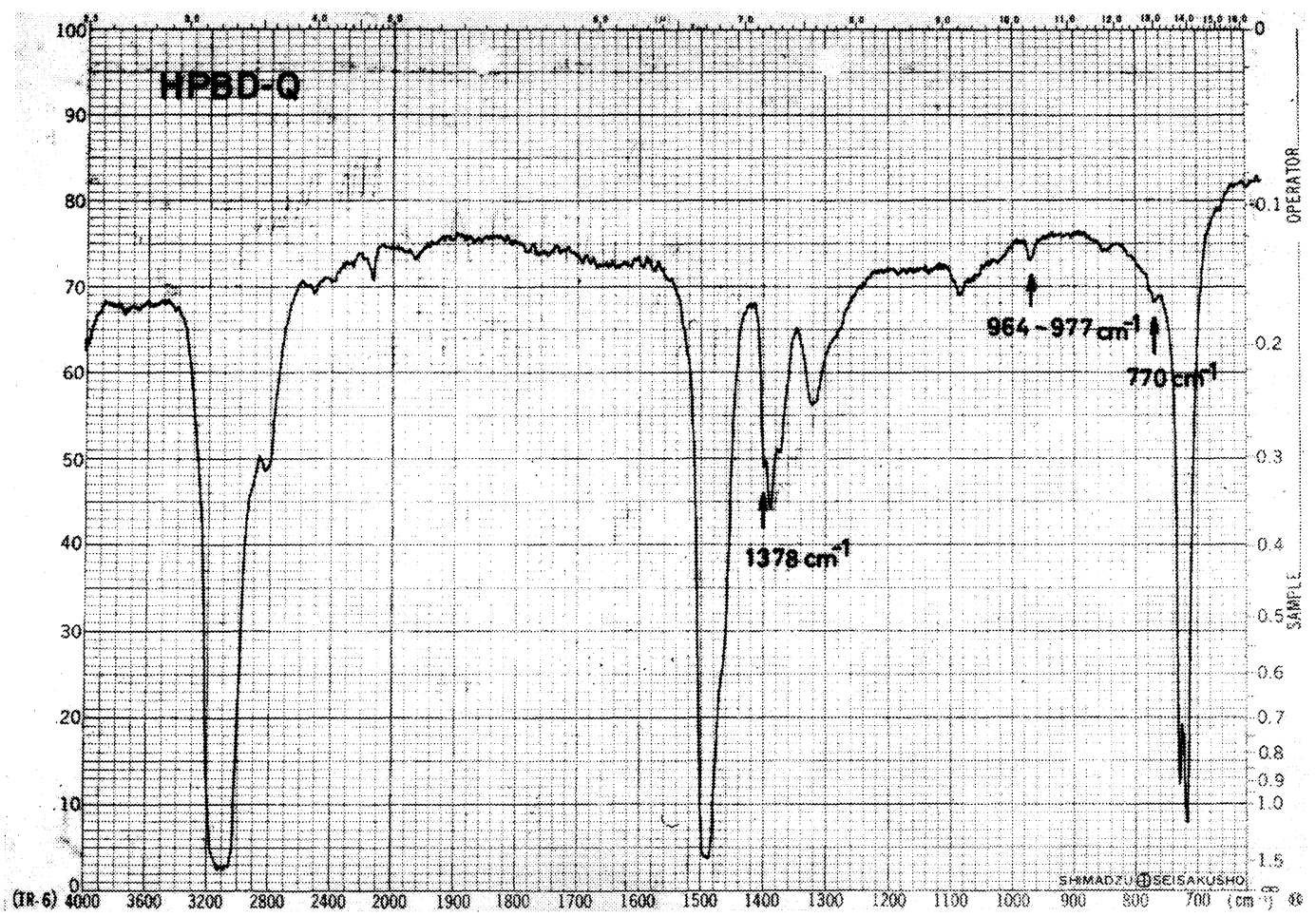

Figure 1. The infrared spectrum of the HPBD-Q film. 
Table I. The number of branches per 100 carbon atoms

\begin{tabular}{lccc}
\hline & Methyl-branch & Ethyl-branch & Butyl-branch \\
\hline HPB & $1.04 \pm 0.05$ & $2.5 \pm 0.2$ & None $^{\mathrm{a}}$ \\
LDPE & $1.02 \pm 0.05$ & Very small $^{\mathrm{b}}$ & $1.34 \pm 0.10$ \\
(UBE B128) & & & \\
\hline
\end{tabular}

a Not recognized in IR spectrum.

b Estimated to be smaller than 0.2.

polymerization of butadiene. Second, a peak, assigned to the $\mathrm{C}-\mathrm{H}$ out-of-plane bending vibration for trans 1-4 double bond appears at about 967 $\mathrm{cm}^{-1}, 7,8$ indicating that the hydrogenation is not complete and some double bonds still remain (a few percent based on the original double bonds ${ }^{3}$ ).

Macroscopic Characteristic. The density of the sample specimens was measured by the floating technique in a water-ethanol mixture. Weight and volume fraction of crystallinities evaluated by using amorphous and crystalline densities of $\rho_{\mathrm{a}}=0.852$ and $\rho_{\mathrm{c}}=1.00 \mathrm{~g} \mathrm{~cm}^{-39}$ are tabulated in Table II. As can be seen from Table II, the degree of crystallinity for quenched and annealed HPB films are a little higher than those for quenched and annealed films of low-density polyethylene, respectively. However, the difference may not cause a significant change in rheo-optical properties.

Differential scanning calorimetry curves for HPBD-Q and HPBD-A films are shown in Figure 2. The heating rate was $10^{\circ} \mathrm{C} \mathrm{min}{ }^{-1}$. As is evident from the figure, the endothermic peak corresponding to the melting of crystals can be observed. The melting point read from the position of the peak in the thermogram is about $104^{\circ} \mathrm{C}$ and $105^{\circ} \mathrm{C}$ for HPBD-Q and HPBD-A films, respectively. These values are

Table II. Density and crystallinity

\begin{tabular}{ccccc}
\hline \multirow{2}{*}{ Sample } & \multicolumn{2}{c}{ Density } & & \multicolumn{2}{c}{ Crystallinity } \\
\cline { 2 - 2 } \cline { 5 - 5 } & $\mathrm{gcm}^{-3}$ & & $X_{\mathrm{w}} / \%$ & $X_{\mathrm{v}} / \%$ \\
\hline HPBD-Q & 0.925 & & 53.3 & 49.6 \\
HPBD-A & 0.933 & & 58.7 & 54.7 \\
LDPE-Q & 0.914 & & 45.8 & 41.9 \\
LDPE-A & 0.925 & & 53.3 & 49.3 \\
\hline
\end{tabular}

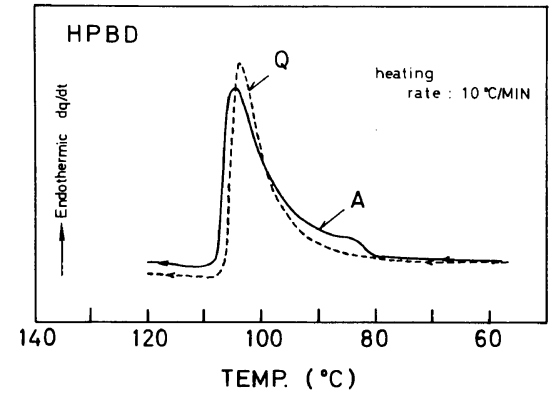

Figure 2. The DSC curves of the HPBD-Q and HPBDA films.

much lower compared with $135^{\circ} \mathrm{C}$ for the highdensity polyethylene, Marlex, ${ }^{10}$ and also with $112^{\circ} \mathrm{C}$ for a typical low-density polyethylene, DYNH. ${ }^{10}$ The melting point of a relatively pure material is described by the van't Hoff equation, ${ }^{11}$

$$
T_{\mathrm{o}}-T_{\mathrm{m}}=\frac{R T_{\mathrm{o}}^{2} X_{2}}{\Delta H_{\mathrm{f}}}
$$

where $T_{\mathrm{o}}(\mathrm{K})$ is the melting point of the $100 \%$ pure material; $T_{\mathrm{m}}(\mathrm{K})$, the melting point of impure material; $R$, the gas constant; $\Delta H_{\mathrm{f}}$, the heat of fusion; and $X_{2}$, the mole fraction of impurities. When we evaluate the mole fraction of impurities in HPB using $T_{0}=410.5 \mathrm{~K}$ and $\Delta H_{\mathrm{f}}=960 \mathrm{cal} \mathrm{mol}^{-1}$ reported by Quinn, Jr. and Mandelkern ${ }^{12}$ for unfractionated polyethylene, we obtain $X_{2}=0.09 \sim 0.10$. This result may suggest that the presence of double bonds and ethyl branches in HPB not only prevents the building up of larger crystals but also compels the organization of crystals having many defects.

$H_{\mathrm{v}}$-light scattering patterns for HPBD-Q and HPBD-A films are shown in Figure 3. The scattering intensity for the Q-film is much weaker than that for 
HPBD
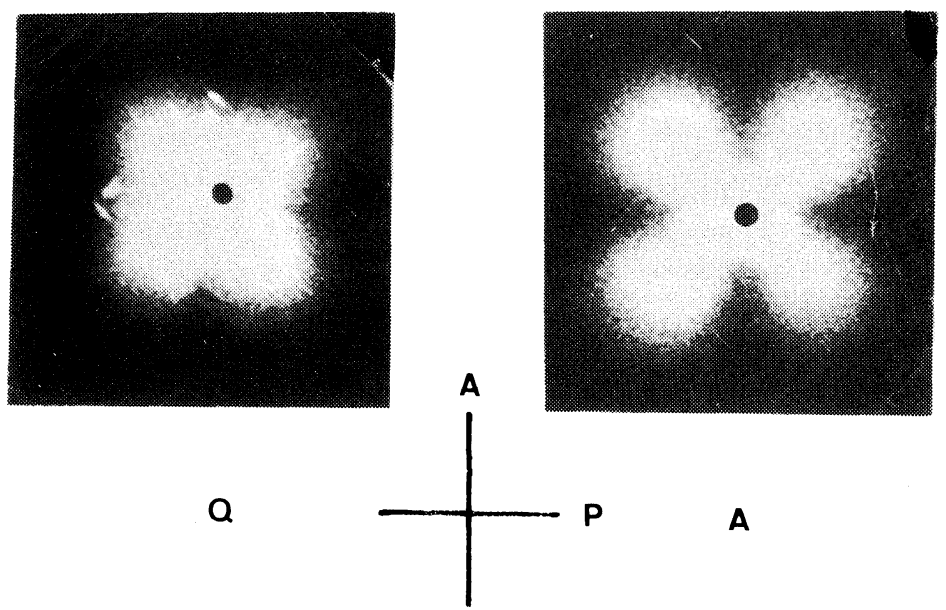

Figure 3. The $H_{\mathrm{v}}$-light scattering patterns of the HPBD-Q and HPBD-A films.

the A-film, when the measuring conditions, such as the exposure time and screen-sample distance required, are taken into account. Both the patterns for the Q- and A-films appear to be of the four-leaf clover type, suggesting the existence of spherulitic structure. The four-leaf clover pattern for the Qfilm, however, is much less clear as compared with that for the A-film and for the bulk-crystallized lowand high-density polyethylene film (quenched and annealed). This fact indicates that the spherulitic structure in the Q-film is considerably disordered or disorganized. The spherulitic structure in the A-film, on the other hand, is well-ordered compared with that in the Q-film, but its order seems to be comparable to or somewhat less than that in polyethylene films melt-crystallized under similar conditions. The average radius of spherulites estimated by the method of Stein and Rhodes ${ }^{13}$ is $5 \sim 10 \mu \mathrm{m}$ for both the Q- and A-films.

\section{RESULTS AND DISCUSSION}

\section{Stress-Strain Cycle}

Figure 4 illustrates stress-strain cycles obtained for HPBD-A and HPBD-Q films at a constant strain rate of $20 \% \mathrm{~min}^{-1}$. HPB films, especially the quenched film (bottom), manifest much better elastic recovery compared to bulk-crystallized polyethylene films.

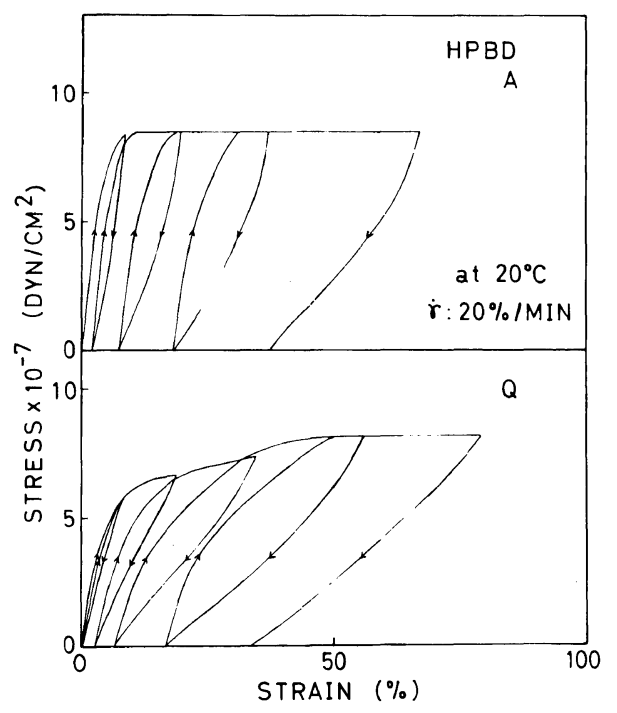

Figure 4. The stress-strain cycles for the HPBD-A (top) and HPBD-Q (bottom) films.

\section{Dynamic Viscoelastic Properties}

The dynamic viscoelastic measurements were carried out at a frequency of $35 \mathrm{~Hz}$ by using a Rheovibron, Model DDV-III. Temperature dependences of the storage Young's modulus $E^{\prime}$, loss modulus $E^{\prime \prime}$, and mechanical loss tangent for HPBD-Q and HPBD-A films are shown in Figure 5. 


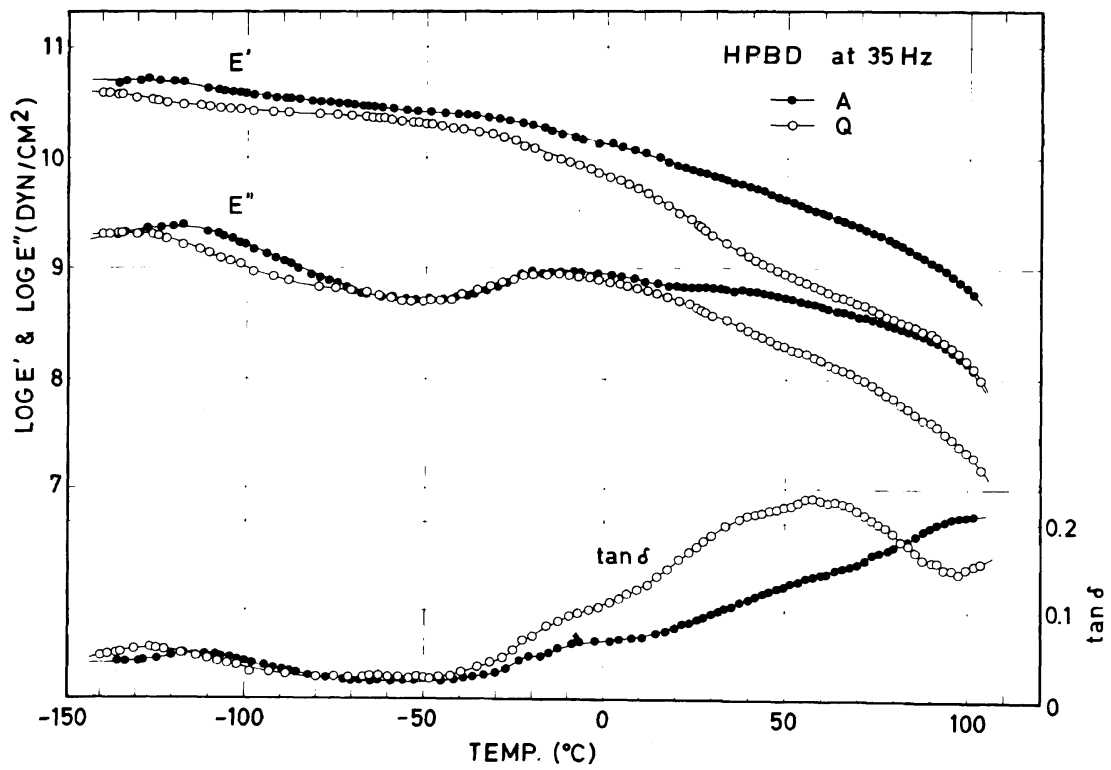

Figure 5. The temperature dependence of the storage Young's modulus $E^{\prime}$, the loss modulus $E^{\prime \prime}$, and the mechanical loss tangent $\tan \delta$ for the HPBD-Q and HPBD-A films at a frequency of $35 \mathrm{~Hz}$.

The alpha, beta, and gamma dispersions are clearly evidenced by maxima (or shoulder) in $E^{\prime \prime}$ and $\tan \delta$ curves as well as by the corresponding decreases in $E^{\prime}$. These dispersions for the HPBD-Q film center at $60^{\circ} \mathrm{C},-10^{\circ} \mathrm{C}$, and $-127^{\circ} \mathrm{C}$, respectively. For the HPBD-A film, on the other hand, the dispersions center at $100^{\circ} \mathrm{C},-10^{\circ} \mathrm{C}$, and $-112^{\circ} \mathrm{C}$, respectively.

As is clear from the figure, the gamma-dispersion peak is shifted to the high-temperature side by annealing. This shift was observed by previous authors for crystals of $n$-alkanes, ${ }^{14}$ single crystals of linear polyethylene, ${ }^{15}$ and melt-crystallized polyethylene. ${ }^{14,15}$ The gamma dispersion for HPB may also be explained by the hypothesis proposed by Sinnott for polyethylene. ${ }^{15}$ According to him, the gamma dispersion is due to the stress-induced reorientation of defects within the lamellae.

The beta dispersion at about $-10^{\circ} \mathrm{C}$ is observed for both the HPBD-Q and HPBD-A films. In general, the beta dispersion can better be observed for branched polyethylene rather than for linear polyethylene. ${ }^{16}$

The alpha dispersion is shifted to the hightemperature side by annealing, as is the gamma dispersion. This shift was observed for meltcrystallized polyethylene by previous investi- gators. ${ }^{14,15,17-19}$ The alpha dispersion depends strongly on the thermal history of the sample employed. ${ }^{14,15,17,19,20}$ The mechanism of the alpha dispersion is discussed in detail elsewhere. ${ }^{15,19}$ In regard to dynamic mechanical properties, HPB is very similar to branched polyethylene.

\section{Stress Relaxation and Birefringence Relaxation}

The stress relaxation and birefringence relaxation were measured at various temperatures ranging from $20^{\circ} \mathrm{C}$ and $80^{\circ} \mathrm{C}$. Several strips, $4 \mathrm{~cm}$ in length and $1 \mathrm{~cm}$ in width, were cut from sample films and used for making the relaxation measurements. At first, the strip was elongated at a constant speed of $1000 \% \min ^{-1}$ to give a strain of $2 \%$, and the variation of stress with time was measured. In Figures 6 and 7, the relaxation modulus at various temperatures is logarithmically plotted against time for HPBD-Q and HPBD-A films, respectively. As is clear from the figures, the relaxation modulus for both the HPBD-Q and HPBD-A films decreases with increasing time. However, such stress relaxation for HPB films, especially the Q-film, is considerably gradual, compared with that for lowand high-density polyethylenes previously ${ }^{20-22}$ observed. 


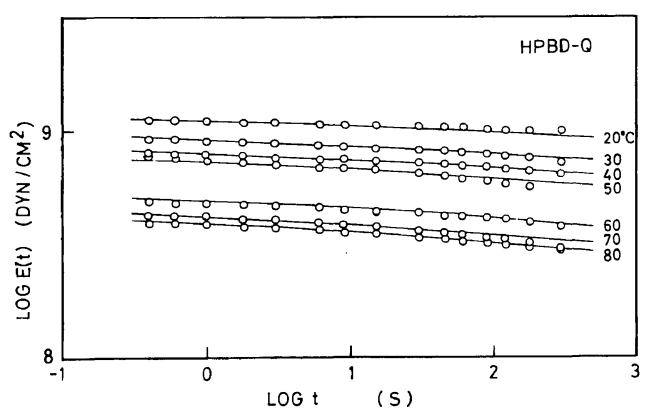

Figure 6. The variation of relaxation modulus $E(t)$ with time $t$ for the HPBD-Q film at various temperatures.

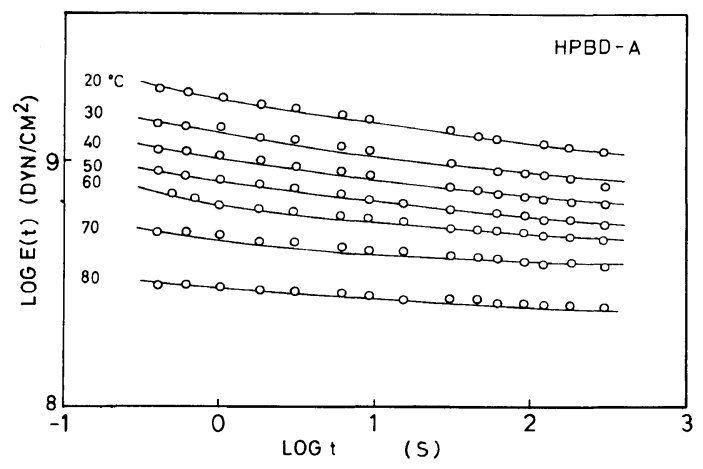

Figure 7. The variation of relaxation modulus $E(t)$ with time $t$ for the HPBD-A film at various temperatures.

The birefringence-relaxation data for the HPBDA film corresponding to the stress-relaxation data shown in Figure 7 are given in Figure 8. The ordinate of this figure is the strain-optical coefficient, $\Delta / \gamma$. As is evident from the figure, the birefringence increases with increasing time. The rates of increase at various temperatures are very similar to those for low-density polyethylene studied previously. ${ }^{21}$ It was found that the birefringence for the HPBD-Q film does not vary with time, though a slight increase in the birefringence was observed for shorter times only at $20^{\circ} \mathrm{C}$.

The time-temperature superposition method was applied to the stress relaxation and birefringence relaxation curves shown in Figures 6, 7, and 8. In order to obtain smooth master curves, not only horizontal but also vertical shifts were required.

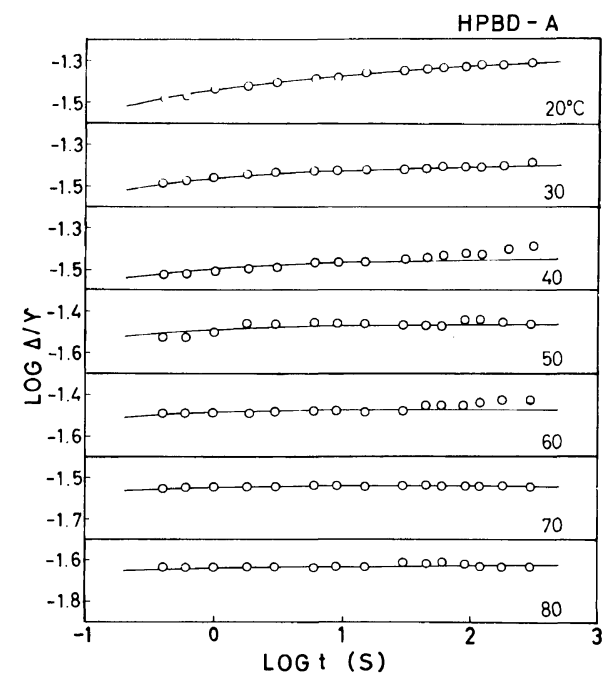

Figure 8. The variation of strain-optical coefficient $\Delta / \gamma$ with time $t$ for the HPBD-A film at various temperatures.

Master curves of $E(t)$ and $\Delta / \gamma(t)$ for the HPBD-Q and HPBD-A films are shown in Figure 9. In the case of $\Delta / \gamma$ for the HPBD-Q film, the timetemperature superposition could not be applied, because $\Delta / \gamma$ is independent of time at temperatures between $30^{\circ} \mathrm{C}$ and $80^{\circ} \mathrm{C}$. So, the master curve of $\Delta / \gamma$ was hypothetically drawn, assuming that $\Delta / \gamma$ curves at various temperatures can be superposed by the same shift factors as those used for $E(t)$. For comparison, master curves of $E(t)$ and $\Delta / \gamma(t)$ obtained previously ${ }^{21}$ for heat-treated film of lowdensity polyethylene, LDPE-H, are also shown with thick solid lines in the same figure. This film of lowdensity polyethylene was prepared as follows. ${ }^{21}$ Commercial pellets, UBE B-128, were first meltpressed at $160^{\circ} \mathrm{C}$ and $30 \mathrm{~kg} \mathrm{~cm}^{-2}$ and then quenched in an ice-water bath. The film thus obtained was heat-treated in an oven at $105^{\circ} \mathrm{C}$ for $45 \mathrm{~min}$.

As is evident from Figure 9, the relaxation moduli for all the samples decrease with reduced time, $t / a_{\mathrm{T}}$, but the relaxation modulus for HPBD-Q film decreases much more gradually as compared with other moduli. The master curve of the relaxation modulus for HPBD-A film is very close to that of LDPE-H film, though the former is slightly lower than the latter.

The master curves of the strain-optical coefficient for HPBD-A and LDPE-H films increase with 
Elastic Recovery of Hydrogenated Polybutadiene

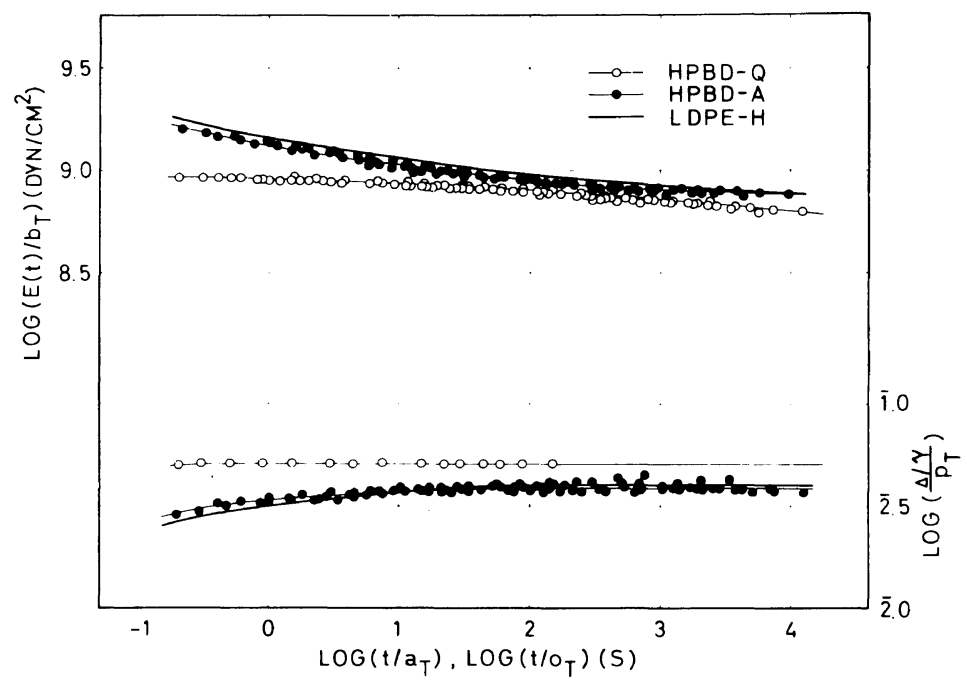

Figure 9. The master curves of relaxation moduli and strain-optical coefficients for the HPBD-Q and HPBD-A films. The solid lines show the master curves for the low-density polyethylene film obtained previously. The reference temperature is $30^{\circ} \mathrm{C}$.

reduced time, $t / o_{\mathrm{T}}$. However, the strain-optical coefficient for HPBD-Q film is almost constant and independent of time. A very slight increase can be seen only at the short-time end.

As shown in Figures 10 and 11, the viscoelastic shift factor $a_{\mathrm{T}}$ and rheo-optical shift factor $o_{\mathrm{T}}$ plotted logarithmically against the reciprocal absolute temperature $1 / T$ give straight lines for HPBD-Q and HPBD-A films. In Figure $10, o_{\mathrm{T}}$ is not included, since the strain-optical coefficients for the HPBD-Q are superposed with the viscoelastic factors, as mentioned above. The activation energies, evaluated from the slope of $a_{\mathrm{T}} v s .1 / T$ and $o_{\mathrm{T}}$ vs. $1 / T$ plots, are summarized in Table III. It is evident from this table that the activation energies are about $30 \mathrm{kcal} \mathrm{mol}^{-1}$. This activation energy is equal to or slightly higher than that for the $\alpha_{1}$ dispersion of the polyethylene. ${ }^{19-21}$

\section{Change in Structure due to Drawing}

In order to clarify the change in structure due to the drawing of HPBD-Q film, the X-ray diffraction, birefringence, and infrared dichroism were measured, and these results have been compared with those for LDPE-Q film.

The X-ray diffraction measurement was carried out with a conventional diffractometer, manufactured by the Shimadzu Corporation (Model VD-1).
The Herman's type orientation functions, ${ }^{23} f_{110}$ and $f_{200}$, are given by

$$
f_{i}=\frac{3\left\langle\cos ^{2} \eta_{i}\right\rangle-1}{2} \quad(i=110,200)
$$

Where $\eta_{i}$ is the angle between the stretching direction and the normal vector of a crystal plane, $i$. The mean square of $\cos \eta_{i},\left\langle\cos ^{2} \eta_{i}\right\rangle$, is estimated from the azimuthal-angle dependencies of diffracted intensities at the Bragg angles, $\theta_{110}$ and $\theta_{200}$ for the 110- and 200-planes $\left(2 \theta_{110} \simeq 21.3^{\circ}, 2 \theta_{200} \simeq 23.5^{\circ}\right)$. The intensities at $\theta_{110}$ and $\theta_{200}$ were corrected by subtracting the background intensity, estimated from the intensities at $2 \theta=12^{\circ}$ and $2 \theta=28^{\circ}$, by the following equations.

$$
I_{110}^{\mathrm{B}}=\frac{I_{2 \theta=28} \cdot\left(2 \theta_{110}-12\right)+I_{2 \theta=12} \cdot\left(28-2 \theta_{110}\right)}{16}
$$

and

$$
I_{200}^{\mathrm{B}}=\frac{I_{2 \theta=28} \cdot\left(2 \theta_{200}-12\right)+I_{2 \theta=12} \cdot\left(28-2 \theta_{200}\right)}{16}
$$

The orientation functions, $f_{\alpha}, f_{\beta}$, and $f_{\varepsilon}$ of the crystal $a-, b$-, and $c$-axes are related to $f_{110}$ and $f_{200}$ by Wilchinsky's equations, ${ }^{24}$ 


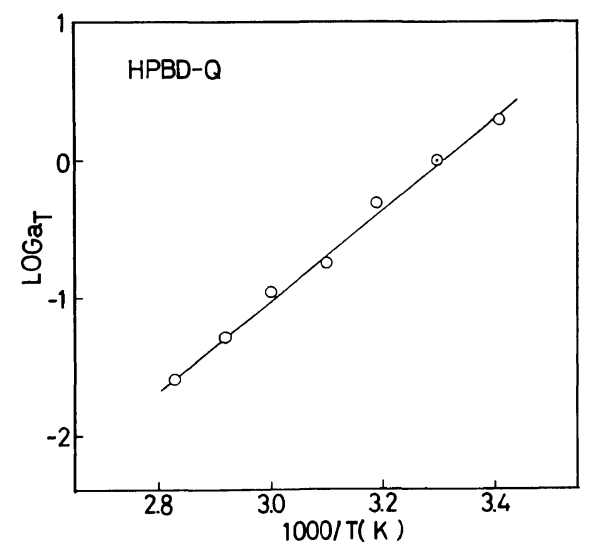

Figure 10. Viscoelastic shift factor, $a_{\mathrm{T}}$ plotted against $1 / T$ for the HPBD-Q film.

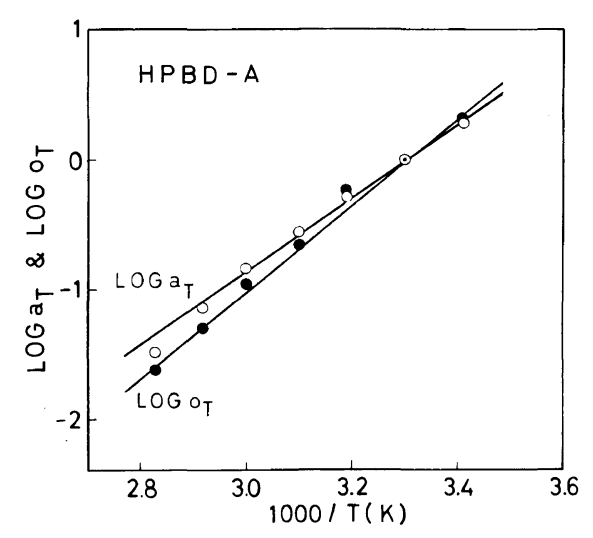

Figure 11. Viscoelastic and rheo-optical shift factors, $a_{\mathrm{T}}$ and $o_{\mathrm{T}}$, plotted against $1 / T$ for the HPBD-A film.

Table III. Activation energies

\begin{tabular}{ccc}
\hline \multirow{2}{*}{ Sample } & \multicolumn{2}{c}{ Activation energy } \\
\cline { 2 - 3 } & \multicolumn{2}{c}{ kcal mol $^{-1}$} \\
\cline { 2 - 3 } & Viscoelastic & Rheo-optical \\
\hline HPBD-Q & 32.1 & \\
HPBD-A & 36.1 & 32.5 \\
LDPE-H $^{\text {a }}$ & 28.4 & 28.4 \\
\hline
\end{tabular}

${ }^{a}$ S. Onogi et al., Polym. J., 7, 467 (1975).

$$
\begin{aligned}
& f_{\alpha}=f_{200} \\
& f_{\beta}=1.445 f_{110}-0.445 f_{200}
\end{aligned}
$$

and

$$
f_{\varepsilon}=-f_{\alpha}-f_{\beta}
$$

In Figure 12, the orientation functions $f_{\alpha}, f_{\beta}, f_{\varepsilon}$ for HPBD-Q film are plotted against the strain $\gamma$ together with those for the LDPE-Q film. These measurements were carried out at room temperature $\left(25^{\circ} \mathrm{C}\right)$. As is evident from the figure, $f_{\alpha}$ and $f_{\beta}$ are negative for both samples, though the strain dependencies of $f$ 's for HPBD-Q and LDPE-Q films are not always the same, especially in the strain region below $20 \%$. For HPBD-Q film, the magnitude of $f_{\alpha}$ is larger than that of $f_{\beta}$, and $f_{\beta}$ is near zero. For the LDPE-Q film, on the other hand, $f_{\alpha}$ and $f_{\beta}$ are very close to each other. In discussing the orientation mechanism of crystallites for LDPE-Q films, the affine deformation of spherulitic structure should be taken into consideration. However, in the case of HPBD-Q films, the affine deformation of spherulitic structure may not affect the orientation mechanism of the crystallites strongly, as is suggested from the experimental results in the stress and birefringence relaxations, i.e., that the time scale of orientation of crystallites is as short as that of the affine deformation of spherulitic structure. In consideration of the above, it may be suggested that the orientation mechanism of crystallites in the lamellae in HPBD-Q film is governed preferentially

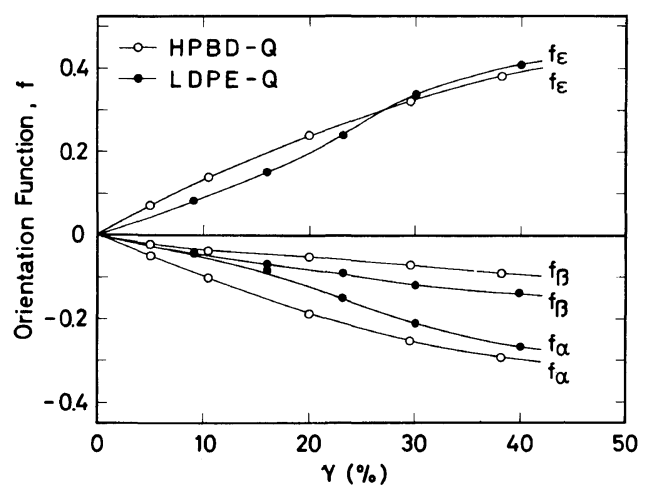

Figure 12. Orientation functions, $f_{\alpha}, f_{\beta}$, and $f_{\varepsilon}$ at the different strains for the HPBD-Q and LDPE-Q films. The measurements are carried out at a room temperature $\left(25^{\circ} \mathrm{C}\right)$. 
by the lamella twisting, but in the LDPE-Q film, mainly by the tilting of crystallites in the lamella.

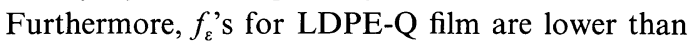
those for HPBD-Q film in the strain region below $20 \%$, indicating that the orientation of crystallites in LDPE-Q film is more difficult than that in HPBD-Q film. This suggests the possibility of the occurrence of slippage of crystallites in addition to the tilting in LDPE-Q film.

Birefringence was measured at different strain levels with a Babinet compensator. It was shown ${ }^{25}$ that the birefringence of crystalline polymers consists of contributions from the amorphous and crystalline phases, $\Delta_{\mathrm{am}}$ and $\Delta_{\mathrm{cr}}$, and form birefringence, $\Delta_{\mathrm{f}}$ as expressed by the equation,

$$
\begin{aligned}
& \Delta_{\text {total }}=\Delta_{\mathrm{am}}+\Delta_{\mathrm{cr}}+\Delta_{\mathrm{f}} \\
& \Delta_{\mathrm{am}}=\left(1-X_{\mathrm{c}}\right) f_{\mathrm{am}} \Delta_{\mathrm{am}}^{\circ}
\end{aligned}
$$

and,

$$
\Delta_{\mathrm{cr}}=X_{\mathrm{c}}\left[\left(n_{a}-n_{c}\right) f_{\alpha}+\left(n_{b}-n_{c}\right) f_{\beta}\right]
$$

where $X_{\mathrm{c}}$ is the volume fraction crystallinity, $n_{a}, n_{b}$ and $n_{c}$ are the refractive indices along the $a-, b$-, and $c$-axes, $f_{\text {am }}$ is the amorphous-orientation function, $\Delta_{\mathrm{am}}^{\circ}$ is the intrinsic birefringence of the amorphous phase. $\Delta_{\mathrm{am}}$ and $\Delta_{\mathrm{cr}}$ were calculated using eq 8 and 10 , neglecting $\Delta_{\mathrm{f}}$, being very small.

In Figure 13, $\Delta_{\text {total }}, \Delta_{\mathrm{cr}}$, and $\Delta_{\mathrm{am}}$ are plotted against strain levels for HPBD-Q and LDPE-Q films. As is evident from the top figure, the greater part of the total birefringence for HPBD-Q film comes from the crystalline phase. But for LDPE-Q film (bottom figure), the birefringence due to the amorphous phase is fairly large, especially at strains above $30 \%$, though the contribution of the crystalline phase is larger than that of the amorphous phase over the entire range of strain level. This result also suggests that the deformation mechanism in HPBD-Q film may differ from that in LDPE-Q film.

Infrared dichroism measurements for HPBD-Q and LDPE-Q films were also carried out at several strain levels, in order to obtain information on the orientation of amorphous chains. The orientation function was determined by the method of Read and Stein, ${ }^{26}$ who investigated the following bands.

$1078 \mathrm{~cm}^{-1}$. This band is assigned to a skeletal $\mathrm{C}-\mathrm{C}$ stretching mode of both trans and gauche conformations in the amorphous phase. ${ }^{27}$ The
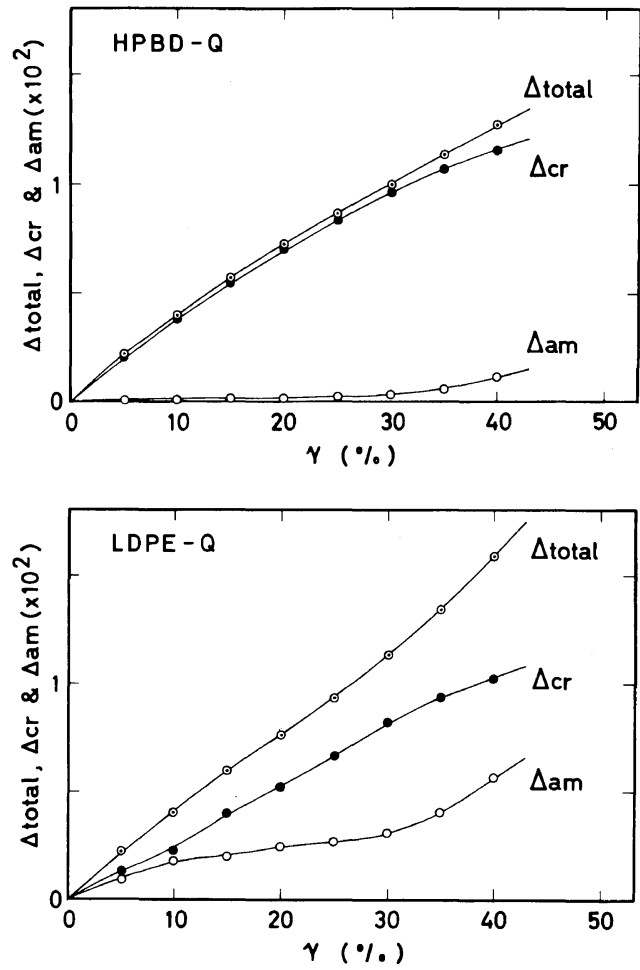

Figure 13. The variation of $\Delta_{\text {total }}, \Delta_{\mathrm{cr}}$, and $\Delta_{\mathrm{am}}$ with the strain for the HPBD-Q (top) and LDPE-Q (bottom) films at a room temperature $\left(25^{\circ} \mathrm{C}\right)$.

transition moment is perpendicular to the chain axis. ${ }^{28}$

$1303 \mathrm{~cm}^{-1}$. This band is assigned to a $\mathrm{CH}_{2}$ twisting mode of amorphous-chain segments in the gauche conformation. ${ }^{27,28}$ A more recent discussion $^{29}$ associates this band with a wagging of $\mathrm{CH}_{2}$ groups in a GTG or GTG' [where G stands for a gauche $(+), T$ for a trans, and $\mathrm{G}^{\prime}$ for a gauche $(-)]$ conformation which is antisymmetrical with respect to the center of the trans bond. This may have a transition moment parallel to the chain axis.

$1352 \mathrm{~cm}^{-1}$. This band is assigned to a $\mathrm{CH}_{2}$ wagging mode of amorphous chain segments in the gauche conformation. ${ }^{27}$ According to Snyder, ${ }^{29}$ this band is due to the wagging of a $\mathrm{CH}_{2}$ flanked by gauche bonds (GG), and its transition moment should be perpendicular to the plane of $\mathrm{CH}_{2}$ groups. $1368 \mathrm{~cm}^{-1}$. Snyder ${ }^{29}$ assigns this band, like the $1303 \mathrm{~cm}^{-1}$ band, to a $\mathrm{CH}_{2}$ wagging in GTG or GTG' conformation; here, the motion is symmetri- 
cal with respect to the center of the bond.

$2016 \mathrm{~cm}^{-1}$. This band is attributed to both the crystalline and amorphous phases, ${ }^{27,30}$ though a more recent paper ${ }^{31}$ has apparently regarded it as a crystalline band. In order to evaluate the orientation function of amorphous-chain segments, separation of the amorphous contribution of the dichroic ratio from the total dichroic ratio measured directly is required. The method of separation is described in a paper by Read and Stein. ${ }^{26}$ To apply this method, it is necessary to obtain rather precise data on the orientation function of the crystal $c$-axis, the extinction coefficients of perfectly oriented crystals, the proportion of the crystalline contribution, and the extinction coefficients for the pallalel and perpendicular polarized infrared. In this study, only the overall orientation function, $f_{2016}^{\text {am }+ \text { cryst }}$, including both the amorphous and crystalline contributions was determined, because data precise enough to evaluate the orientation function of amorphous chain segments could not be obtained. Both the crystalline and amorphous components have a transition moment parallel to the $c$-axis. ${ }^{27,30}$

Orientation functions are related to dichroic ratios as follows.

$$
\begin{array}{r}
f_{1303}^{\mathrm{am}}=\frac{D_{1303}-1}{D_{1303}+2} \\
f_{1352}^{\mathrm{am}}=\frac{D_{1352}-1}{D_{1352}+2} \\
f_{1368}^{\mathrm{am}}=\frac{D_{1368}-1}{D_{1368}+2} \\
f_{2016}^{\mathrm{am}+\text { cryst }}=\frac{D_{2016}-1}{D_{2016}+2} \\
f_{1078}^{\text {am }}=-2 \frac{D_{1078}-1}{D_{1078}+2}
\end{array}
$$

where the dichroic ratio for a given band is defined by the ratio of the pallalel extinction coefficient $\varepsilon_{\|}$to the perpendicular $\varepsilon_{\perp}$.

In Figure 14, the orientation functions of amorphous-chain segments with different conformations are plotted against several strain levels. The overall amorphous-orientation function obtained by the birefringence measurements is also plotted in

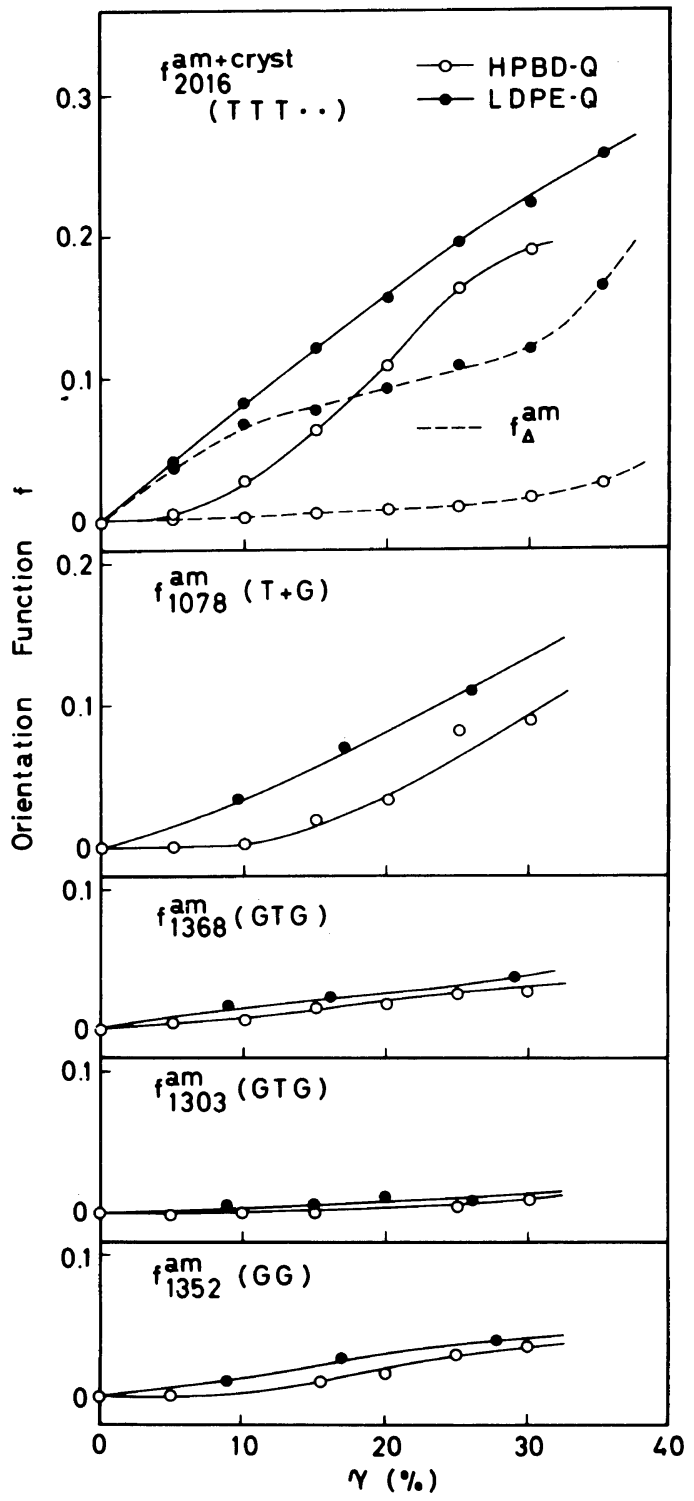

Figure 14. The variation of $f_{2016}^{\mathrm{am}+\mathrm{cryst}}, f_{1078}^{\mathrm{am}}, f_{1368}^{\mathrm{am}}, f_{1303}^{\mathrm{am}}$, $f_{1352}^{\mathrm{am}}$ and $f_{\Delta}^{\mathrm{am}}$ with the strain for the HPBD-Q and LDPE-Q films at a room temperature $\left(25^{\circ} \mathrm{C}\right)$.

the same figure, and is determined by eq 16 derived from eq 9.

$$
f_{\mathrm{am}}=\frac{\Delta_{\mathrm{total}}-\Delta_{\mathrm{cr}}}{\left(1-X_{\mathrm{c}}\right) \Delta_{\mathrm{am}}^{\circ}}
$$

$\Delta_{\mathrm{am}}^{\circ}$ in this equation was estimated as the summation of bond polarizabilities by Bunn and 
Daubeny. ${ }^{32}$ As is evident from Figure $14, f_{2016}^{\mathrm{am}+\text { cryst }}$ is largest and $f_{1078}^{\text {am }}$ and so forth follow, suggesting that the sequence of trans-rich conformation does orient more than that of gauche-rich conformation. All the orientation functions of the amorphous-chain segments increase monotonically with increasing strain. However, $f_{\Delta}^{\mathrm{am}}$ 's increase rapidly above $30 \%$ strain. An increase in the crystallinity by the stretching may bring the increase in $f_{\Delta}^{\mathrm{am}}$, since $f_{\Delta}^{\mathrm{am}}$ is estimated on the assumption of constant crystallinity under the strains covered. However, such an increase in crystallinity cannot be expected at all with elongations as small as $30 \%$; the crystallinity may rather decrease due to possible breaking of the spherulites. A probable explanation of a rapid increase in $f_{\Delta}^{\text {am }}$ above $30 \%$ strain is that amorphous chains become trans-rich rapidly above $30 \%$ strain.

Under a small strain $(<20 \%)$, any amorphous orientation for HPB film is smaller than that for LDPE film, indicating that amorphous chains in the HPB film orient much less compared with those in LDPE film.

\section{CONCLUSION}

The highly elastic properties of HPBD-Q film arises from reversible crystallite orientation which can occur even under relatively large strains. This reversible orientation is due to a loose spherulitic structure, as is shown by the $H_{\mathrm{v}}$-light scattering pattern. The crystallite orientation is governed mostly by the twisting mechanism, as is shown by the X-ray diffraction data. The time scale of the crystallite orientation in HPBD-Q film should be short, as is shown by the fact that the birefringence relaxes very rapidly.

On the other hand, when the LDPE-Q film is subjected to a strain, crystallites orient slowly in the stretching direction, as is shown by birefringencerelaxation measurements. Moreover, in the case of large deformations, crystallites cannot orient without the spherulites breaking. This will cause irreversible deformation.

Acknowledgement. The authors wish to thank Prof. William W. Graessley for providing the hydrogenated polybutadiene samples. This study was supported partly by a Grant-in-Aid for Scientific Research from the Ministry of Education, Science and Culture (No. 365316).

\section{REFERENCES}

1. H. L. Hsieh, J. Polym. Sci., A, 3, 153 (1965).

2. J. C. Falk, J. Polym. Sci., A-1, 9, 2617 (1971); Makromol. Chem., 160, 291 (1972).

3. W. W. Graessley, private communication.

4. L. H. Cross, R. B. Richards, and H. A. Willis, Discuss. Faraday Soc., 9, 235 (1950).

5. H. L. McMurry and V. Thornton, Anal. Chem., 24, 318 (1952).

6. K. Shirayama, Kagaku, Zokan, 43, 131 (1970) ("Kobunshi no Characterization to Bussei"); E. J. Slowinski, Jr., H. Walter, and R. L. Miller, J. Polym. Sci., 19, 353 (1956); M. Rohmer, Z. Anal. Chem., 170, 147 (1959); A. H. Willbourn, J. Polym. Sci., 34, 569 (1959).

7. R. S. Silas, J. Yates, and V. Thornton, Anal. Chem., 31, 529 (1959).

8. J. L. Binder, J. Polym. Sci., A, 3, 1587 (1965).

9. C. W. Bunn, Trans. Faraday Soc., 35, 482 (1939).

10. B. Ke, J. Polym. Sci., 42, 15 (1960).

11. E. M. Barrall and J. F. Johnson, "Thermal Characterization Techniques," P. E. Slada, Jr. and L. T. Jenkins, Ed., Marcel Dekker, Inc., New York, N.Y., 1970, p 28.

12. F. A. Quinn. Jr. and L. Mandelkern, J. Am. Chem. Soc., 80, 3178 (1958).

13. R. S. Stein and M. B. Rhodes, J. Appl. Phys., 31, 1873 (1960).

14. K. H. Illers, Rheol. Acta, 3, 202 (1964).

15. K. M. Sinnott, J. Appl. Phys., 37, 3385 (1966).

16. R. F. Boyer, Rubber Rev., 36, 1303 (1963).

17. S. Iwayanagi, Rep. Prog. Polym. Phys. Jpn., 5, 135 (1962).

18. K. M. Sinnott, J. Polym. Sci., B, 3, 945 (1965).

19. A. Tanaka, E. P. Chang, B. Delf, I. Kimura, and R. S. Stein, J. Polym. Sci., Polym. Phys. Ed., 11, 1891 (1973).

20. S. Onogi, T. Asada, Y. Fukui, and T. Fujisawa, $J$. Polym. Sci., A-2, 5, 1067 (1967).

21. S. Onogi, A. Tanaka, Y. Ishikawa, and T. Igarashi, Polym. J., 7, 467 (1975).

22. Y. Fukui, T. Sato, M. Ushirokawa, T. Asada, and S. Onogi, J. Polym. Sci., A-2, 8, 1195 (1970).

23. J. J. Hermans, P. Hermans, D. Vermaas, and A. Weidinger, Recl. Trav. Chim., Pays-Bas, 65, 427 (1946).

24. Z. W. Wilchinsky, J. Appl. Phys., 31, 1969 (1960).

25. R. S. Stein, J. Polym. Sci., 31, 327 (1958).

26. B. E. Read and R. S. Stein, Macromolecules, 1, 116 (1968).

27. S. Krimm, Fortschr. Hochpolym.-Forsch., 2, 51 (1960).

28. J. R. Nielsen and R. F. Holland, J. Mol. Spectrosc., 6, 394 (1961).

29. R. G. Snyder, Polym. Prepr., Am. Chem. Soc., Dir. Polym. Chem., 8, 1115 (1967); J. Chem. Phys., 47, 
A. TANAKa, Y. Saihara, and S. ONOGI

1316 (1967).

30. J. R. Nielsen and A. H. Woollett, J. Chem. Phys., 26, 1391 (1957).

31. J. L. Koenig, S. W. Cornell, and D. E. Witenhafer, J.
Polym. Sci., A-2, 5, 301 (1967).

32. C. W. Bunn and Rde P. Daubeny, Trans. Faraday Soc., 50, 1173 (1954). 